\title{
Liquid Cell Crystallization and In-situ Imaging of Thiamethoxam by Helium Ion Microscopy
}

\author{
Alison Pawlicki ${ }^{1}$, Matt Bruch ${ }^{2}$, Anton Ievlev ${ }^{2}$, Jeff Fowler ${ }^{3}$, Olga Ovchinnikova $^{2}$, Alex Belianinov ${ }^{2}$ \\ ${ }^{1}$ Bredesen Center, University of Tennessee, Knoxville, TN, USA \\ ${ }^{2}$ Center for Nanophase Materials Science, Oak Ridge National Laboratory, Oak Ridge, TN, USA \\ ${ }^{3}$ Syngenta Crop Protection, Greensboro NC, USA
}

Pest insect control, an essential component of crop protection and public health, has evolved over a recorded history of three millennia. [1,2] Starting with simple elements, like sulfur, with first recorded use in $1000 \mathrm{BC}$, the practice continues to evolve with more complex synthetically designed insecticides. With each new chemical class, resistant strains of insects soon limit their effectiveness.

Neonicotinoids have been extensively utilized; as the only major new class of insecticides developed in the last four decades. [3] Thiamethoxam, a neonicotinoid, is a member of a major class of insecticides, with outstanding potency and systemic action for crop protection against piercing-sucking pests, which are highly effective for flea control on cats and dogs. These compounds have generally low toxicity to mammals (acute and chronic), birds, and fish.

Common industrial uses of thiamethoxam are to make foliar sprays of dilute aqueous solutions and to coat seeds with the active compound as well as binders, fillers, pigments, and other active ingredients. Amongst the practical challenges are the physical stability of the concentrated products, which typically comprise crystal suspensions, optimization of the products to allow efficient application to seeds, and achieving acceptable handling properties of the coated seeds during industrial-volume packing and transportation through elevators, conveyers, and planters. [4, 5] A key issue slowing the optimization of these products is limited knowledge of the crystallization dynamics of neonicotinoids from solution, and how these process can be altered by additives and agents.

In this work, we utilize a Helium Ion Microscope (HIM), in conjunction with a Protochips liquid cell, to crystallize and image thiamethoxam in-situ. Our HIM, and Scanning Electron Microscopy (SEM) results, shown in Figure 1(a-h), illustrate the growth and morphology of the thiamethoxam crystals at different He+ exposure doses, which is markedly different from what has been typically observed. Energy-dispersive X-ray spectroscopy (EDS) results, Figure 2(a-d) confirm the presence of the thiamethoxam on the liquid cell membrane. While the exact details of the radiolysis driven mechanism behind the formation of thiamethoxam, and its non-standard morphology remain uncertain, this imaging study illustrates the HIM ability to image and induce the crystallization phenomena of soft materials in liquid environments; and sheds light onto the processes involved [7].

\section{References:}

[1] Ware GW. "The Pesticide Book" (Thomson Publ. Fresno, CA ) 418 pp.

[2] Casida JE, Quistad GB. Annual Review of Entomology 43 (1998), p. 1.

[3] Tomizawa M., Casida J. E., Annual Reviews of Pharmacology and Toxicology 45 (2005), p.

247.

[4] Robani, H., HortTechnology 4, (1994), p. 104. 
[5] Halecky, A.; Ren, N.; Lu, J.; Wang, J.; Lockwood, F., Pesticide Formulation and Delivery Systems: 36th Volume, Emerging Trends Building on a Solid Foundation, ASTM STP1595 (2016) p. 183.

[6] Cho, W., Fowler, J.D. and Furst, E.M., Langmuir, 28, (2012), p. 6013.

[7]. The HIM imaging, image analytics and simulations portion of this research was conducted at the Center for Nanophase Materials Sciences, which is a DOE Office of Science User Facility. This research was funded by the Center for Nanophase Materials Sciences, which is a U. S. Department of Energy Office of Science User Facility.
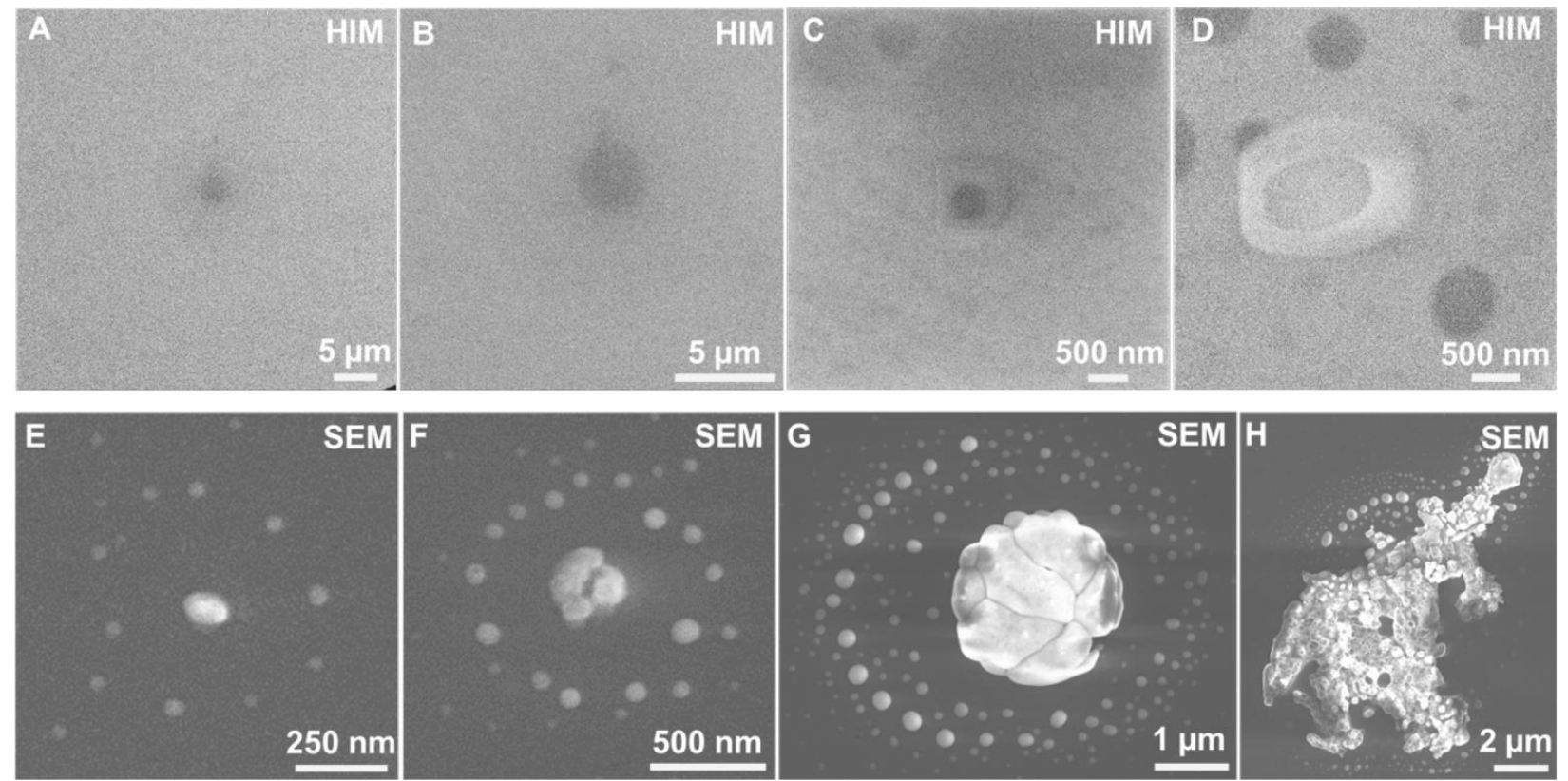

Figure 1. Helium Ion and Secondary Electron images displaying initial to advanced growth on the Si3N4 membrane that was initiated by the He+ beam. A-D) Series of HIM images showing growth, as it occurs, within the liquid cell though the Si3N4 membrane. E-H) SEM images of the underside of the Si3N4 membrane showing the products of the He+ beam induce growth and appear to be nucleated into an amorphous state.
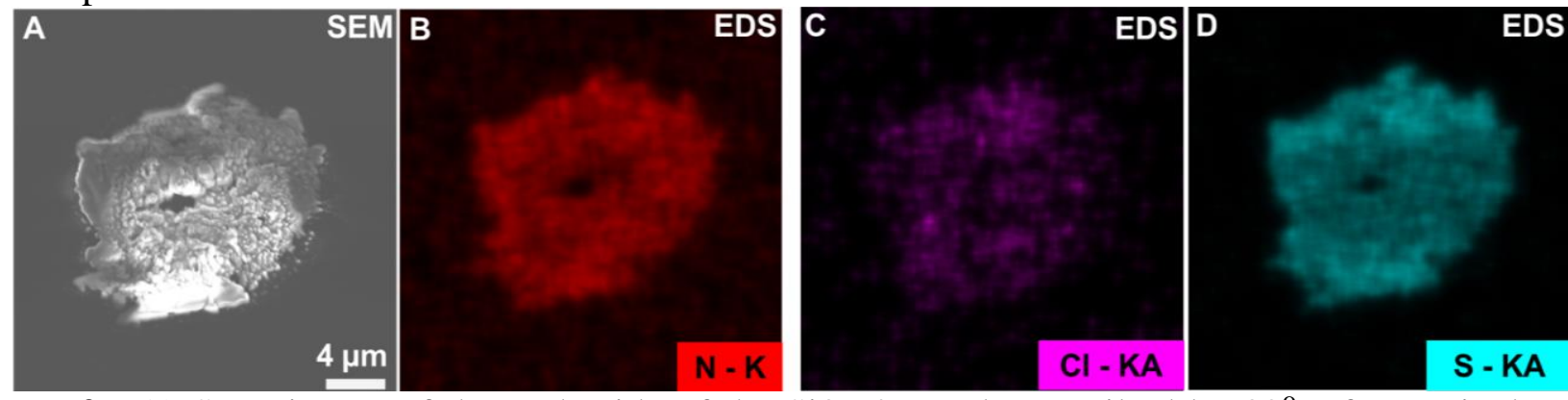

Figure 2. A) SEM image of the underside of the Si3N4 membrane, tilted by $30^{\circ}$, of a particular He+ induced growth feature. B-D) EDS confirms the chemical identity of these growth as TMX. 